OPEN ACCESS

Edited by:

Caroline Mauvezin Institut d'Investigacio Biomedica de Bellvitge (IDIBELL), Spain

Reviewed by: Rafal Ciosk University of Oslo, Norway Shaoli Song,

Fudan University, China

*Correspondence: Kathrin Thedieck kathrin.thedieck@uibk.ac.at; k.thedieck@umcg.nl; kathrin.thedieck@uni-oldenburg.de

Specialty section:

This article was submitted to Cell Growth and Division,

a section of the journal Frontiers in Cell and Developmental

Biology

Received: 02 August 2021 Accepted: 05 October 2021 Published: 29 October 2021

Citation

Rehbein U, Prentzell MT, Cadena Sandoval M, Heberle AM, Henske EP, Opitz CA and Thedieck K (2021) The TSC Complex-mTORC1

Axis: From Lysosomes to Stress

Granules and Back.

Front. Cell Dev. Biol. 9:751892. doi: 10.3389/fcell.2021.751892

\section{The TSC Complex-mTORC1 Axis: From Lysosomes to Stress Granules and Back}

\author{
Ulrike Rehbein', Mirja Tamara Prentzell2,3, Marti Cadena Sandoval1,4, \\ Alexander Martin Heberle ${ }^{1,4}$, Elizabeth P. Henske5, Christiane A. Opitz ${ }^{2,6}$ and \\ Kathrin Thedieck ${ }^{1,4,7 *}$
}

\begin{abstract}
1 Laboratory for Metabolic Signaling, Institute of Biochemistry, Center for Molecular Biosciences Innsbruck, University of Innsbruck, Innsbruck, Austria, ${ }^{2}$ Brain Cancer Metabolism Group, German Consortium of Translational Cancer Research (DKTK) \& German Cancer Research Center (DKFZ), Heidelberg, Germany, ${ }^{3}$ Faculty of Bioscience, Heidelberg University, Heidelberg, Germany, ${ }^{4}$ Section Systems Medicine of Metabolism and Signaling, Department of Pediatrics, University of Groningen and University Medical Center Groningen, Groningen, Netherlands, ${ }^{5}$ Pulmonary and Critical Care Medicine, Department of Medicine, Brigham and Women's Hospital, Harvard Medical School, Boston, MA, United States,

${ }^{6}$ Department of Neurology, National Center for Tumor Diseases, University Hospital Heidelberg, Heidelberg, Germany, ${ }^{7}$ Department for Neuroscience, School of Medicine and Health Sciences, Carl von Ossietzky University Oldenburg, Oldenburg, Germany
\end{abstract}

The tuberous sclerosis protein complex (TSC complex) is a key integrator of metabolic signals and cellular stress. In response to nutrient shortage and stresses, the TSC complex inhibits the mechanistic target of rapamycin complex 1 (mTORC1) at the lysosomes. mTORC1 is also inhibited by stress granules (SGs), RNA-protein assemblies that dissociate mTORC1. The mechanisms of lysosome and SG recruitment of mTORC1 are well studied. In contrast, molecular details on lysosomal recruitment of the TSC complex have emerged only recently. The TSC complex subunit 1 (TSC1) binds Iysosomes via phosphatidylinositol-3,5-bisphosphate [PI(3,5)P2]. The SG assembly factors 1 and 2 (G3BP1/2) have an unexpected lysosomal function in recruiting TSC2 when SGs are absent. In addition, high density lipoprotein binding protein (HDLBP, also named Vigilin) recruits TSC2 to SGs under stress. In this mini-review, we integrate the molecular mechanisms of lysosome and SG recruitment of the TSC complex. We discuss their interplay in the context of cell proliferation and migration in cancer and in the clinical manifestations of tuberous sclerosis complex disease (TSC) and lymphangioleiomyomatosis (LAM).

Keywords: TSC complex, mTORC1 (mechanistic target of rapamycin complex 1), HDLBP, lysosomes, stress granules (SG), autophagy, lymphangioleiomyomatosis (LAM), G3BP1 (G3BP stress granule assembly factor 1)

\section{INTRODUCTION}

For cellular growth and survival, cells have to tightly balance their metabolism to adapt to nutritional changes and environmental stressors. The TSC complex (tuberous sclerosis protein complex) constitutes a key integrator of nutrient and stress signals (Huang and Manning, 2008; Demetriades et al., 2016; Liu and Sabatini, 2020), which adapts cellular metabolism to 
environmental conditions by suppressing the anabolic master regulator mTORC1 (mechanistic target of rapamycin complex 1; Mossmann et al., 2018; Tee, 2018; Kim and Guan, 2019; Hoxhaj and Manning, 2020; Liu and Sabatini, 2020). mTORC1 is an evolutionary highly conserved multi-protein complex. Apart from the MTOR kinase itself, mTORC1 contains the complexspecific interaction partners RPTOR (regulatory associated protein of MTOR complex 1) and AKT1S1 (AKT1 substrate 1) (Yip et al., 2010; Yang et al., 2013; Chao and Avruch, 2019). The TSC complex-mTORC1 axis translates nutrient and stress signals into tightly orchestrated cellular responses that impinge on anabolic processes including translation, as well as catabolic processes such as autophagy (Liu and Sabatini, 2020). Disturbances of the TSC complex lead to mTORC1 hyperactivation and have been linked to diseases including cancer and the clinical manifestations of tuberous sclerosis complex disease (TSC), which are both characterized by cellular overgrowth and aberrant migration (Orlova and Crino, 2010; Borkowska et al., 2011; Curatolo et al., 2015; Henske et al., 2016; Condon and Sabatini, 2019; Jozwiak et al., 2019). Lysosomes are widely recognized as the major signaling platform at which the TSC complex inhibits mTORC1. Also other inhibitory cues such as the RRAG GTPases (Ras related GTP binding proteins) and AMPK (AMP-activated protein kinase) suppress mTORC1 at lysosomes [reviewed in detail by Oakhill et al. (2010); Kim and Guan (2019); Gonzalez et al. (2020); Liu and Sabatini (2020); Fernandes and Demetriades (2021)]. A growing body of evidence shows that stress granules (SGs) constitute a nonmembranous compartment at which mTORC1 is inhibited under stress through several mechanisms (Takahara and Maeda, 2012; Thedieck et al., 2013; Wippich et al., 2013; Ramiscal et al., 2015; Lastres-Becker et al., 2016; Pla-Martin et al., 2020; Mediani et al., 2021). Whereas the molecular machinery mediating the recruitment and regulation of mTORC1 at lysosomes (RabanalRuiz and Korolchuk, 2018; Condon and Sabatini, 2019; Kim and Guan, 2019) or SGs (Takahara and Maeda, 2012; Thedieck et al., 2013; Wippich et al., 2013; Mediani et al., 2021) has been investigated in much detail, recent studies shed light on the mechanisms tethering the TSC complex to lysosomes (Fitzian et al., 2021; Prentzell et al., 2021) and to SGs (Kosmas et al., 2021). In this mini-review we summarize the latest findings focusing on the interplay of the TSC complex with SGs and lysosomes. We discuss the impact of this crosstalk in the context of TSC, lymphangioleiomyomatosis (LAM) and cancer.

\section{MAIN TEXT}

\section{The Lysosomal TSC Complex and SGs Inhibit mTORC1}

The TSC multiprotein complex consists of TSC complex subunit 1 (TSC1), TSC2, and TBC1 domain family member 7 (TBC1D7) (Dibble et al., 2012). The three subunits assemble with a 2:2:1 stoichiometry (Dibble et al., 2012; Ramlaul et al., 2021; Yang et al., 2021). The coiled-coil domains of two TSC1 proteins intertwine in parallel in a double-helix bundle that interacts via several sites with the TSC2 dimer (Ramlaul et al., 2021; Yang et al., 2021). The two TSC2 molecules interact via their dimerization domains in an antiparallel manner, allowing the catalytic pockets of the GAP (GTPase-activating protein) domains to face outward of the TSC complex (Yang et al., 2021). This asymmetric TSC1-TSC2 complex binds a single TBC1D7 molecule via association with one C-terminus in the TSC1 dimer.

In healthy cells, the TSC complex integrates signals from multiple growth factor pathways (Huang and Manning, 2008), as well as nutrient sufficiency and cellular stresses (Demetriades et al., 2014, 2016; Menon et al., 2014; Plescher et al., 2015; Carroll et al., 2016). In response to growth factors, including insulin, the AKT serine/threonine kinase (AKT) phosphorylates TSC2 and inhibits the TSC complex (Inoki et al., 2002; Manning et al., 2002; Potter et al., 2002). Apart from the activation of mTORC1, insulin-AKT signaling enhances a TSC2-independent function of TSC1 in cytostatic and prometastatic TGFB (transforming growth factor beta)-Smad2/3 (SMAD family member 2/3) signaling (Thien et al., 2015). Like AKT, also WNT (Wnt family member) (Inoki et al., 2006) and MAPK (mitogen-activated protein kinase) (Ma et al., 2005) signaling suppress TSC2 via RPS6KA1 (ribosomal protein S6 kinase A1) (Roux et al., 2004) and GSK3B (glycogen synthase kinase 3 beta), respectively. In contrast, phosphorylation of TSC2 by AMPK activates the TSC complex and inhibits mTORC1 (Inoki et al., 2003b). When growth factor signals are low, the TSC complex translocates to the lysosomal surface (Menon et al., 2014). Similarly, deprivation of all amino acids (Demetriades et al., 2014) or of arginine alone (Carroll et al., 2016) as well as hyperosmotic stress, hypoxia, $\mathrm{pH}$ stress and 2-DeoxyD-glucose (Plescher et al., 2015; Demetriades et al., 2016) enhance the lysosomal association of the TSC complex. The TSC complex acts as a GAP that inhibits the small GTPase RHEB (RAS homolog-mTORC1 binding) by enhancing the conversion of RHEB's GTP-bound state to the GDP-bound state (Inoki et al., 2003a; Tee et al., 2003; Zhang et al., 2003). GTP-bound RHEB activates mTORC1, and the TSC complex suppresses mTORC1 upon growth factor shortage, nutrient deprivation, and other stresses (Huang and Manning, 2008; Plescher et al., 2015; Demetriades et al., 2016; Fernandes and Demetriades, 2021). Conversely, growth factor and nutrient sufficiency reduce the amount of lysosomal TSC complex (Demetriades et al., 2014; Menon et al., 2014; Carroll et al., 2016), increasing the abundance of RHEB-GTP and activating mTORC1 (Zhang et al., 2003; Menon et al., 2014). MTOR directly binds to RHEB-GTP, which causes a conformational change in the active site of the MTOR kinase domain (Yang et al., 2017). This allows mTORC1 to bind and phosphorylate its multiple substrates (Liu and Sabatini, 2020). Among them are EIF4EBP1 (eukaryotic translation initiation factor 4E binding protein 1) and RPS6KB1 (ribosomal protein S6 kinase B1), whose phosphorylation by mTORC1 enhances cap-dependent translation, and ULK1 (unc-51 like autophagy activating kinase 1) via which mTORC1 suppresses autophagy (Liu and Sabatini, 2020; Figure 1).

Beyond the lysosomal TSC complex, mTORC1 inhibition under stress is also mediated by SGs, cytoplasmic protein-RNA 


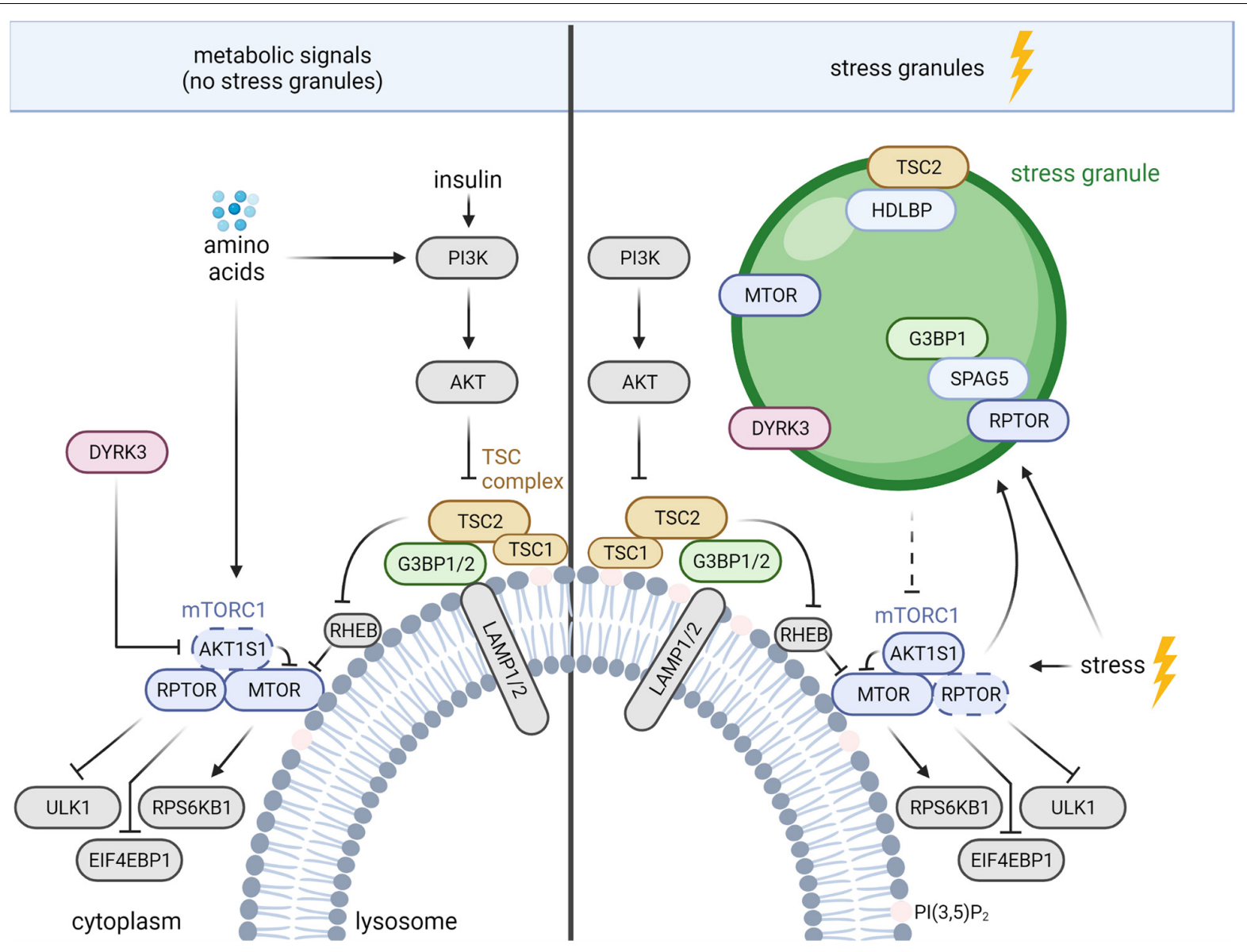

FIGURE 1 | TSC complex-mTORC1 signaling during nutrient sufficiency and stress granule (SG) formation. The mechanisms are described in the text. AKT, AKT serine/threonine kinase; AKT1S1, AKT substrate 1; DYRK, dual specificity tyrosine phosphorylation regulated kinase 3; EIF4EBP1, eukaryotic translation initiation factor $4 \mathrm{E}$ binding protein 1; G3BP 1/2, stress granule assembly factor 1/2; HDLBP, high density lipoprotein binding protein; LAMP1/2, lysosomal associated membrane proteins 1/2; MTOR, mechanistic target of rapamycin kinase; PI3K, phosphoinositide 3-kinases; $\mathrm{PI}(3,5) \mathrm{P}_{2}$, phosphatidylinositol-3,5-bisphosphate; RHEB, RAS homolog-mTORC1 binding; RPS6KB1, ribosomal protein S6 kinase B1; RPTOR, regulatory associated protein of MTOR complex 1; SPAG5, sperm associated antigen 5; TSC1/2, TSC complex subunit 1/2; ULK1, unc-51 like autophagy activating kinase 1. Dashed arrow, inhibition via disassembly of mTORC1.

assemblies formed upon stress-induced inhibition of translation (Takahara and Maeda, 2012; Thedieck et al., 2013; Wippich et al., 2013; Heberle et al., 2015; Mediani et al., 2021). SGs constitute a dynamic non-membranous compartment that sorts mRNAs for maintenance or decay (Advani and Ivanov, 2019), controls signaling networks (Kedersha et al., 2013; Heberle et al., 2015), and promotes survival under stress (Kim et al., 2005; Arimoto et al., 2008; Tsai and Wei, 2010; Thedieck et al., 2013; Park et al., 2020). A variety of stress signals promote SG assembly via mechanisms associated with stalled translation [reviewed in detail by Alberti and Dormann (2019); Hofmann et al. (2021)]. The best described regulators of SG assembly are the eukaryotic translation initiation factor 2 subunit alpha (EIF2S1) kinases (Anderson et al., 2015), which inhibit EIF2S1 to diminish global cap-dependent translation (Holcik, 2015). The release of monosomal mRNAs enables the recruitment of RNA-binding proteins, such as the G3BP stress granule assembly factors 1 and 2 (G3BP1/2 or G3BPs), leading to SG assembly (Anderson et al., 2015; Panas et al., 2016). Pbp1, the yeast ortholog of Ataxin-2, acts under stress to recruit yeast RPTOR (Kog1) and MTOR (Tor1) to SGs (Takahara and Maeda, 2012). SGs also sequester MTOR in mammalian cells, but the recruiting protein remains unknown (Wippich et al., 2013; Figure 1). mTORC1 inhibition by SGs in mammalian cells is mediated by the sperm associated antigen 5 (SPAG5, also known as astrin) that recruits the mTORC1 specific scaffold protein RPTOR to SGs, and disassembles mTORC1 (Thedieck et al., 2013). In addition, SGs regulate mTORC1 via the dual specificity tyrosine phosphorylation regulated kinase 3 (DYRK3) (Wippich et al., 2013; Mediani et al., 2021; Figure 1). Under non-stressed conditions, cytosolic DYRK3 phosphorylates and represses mTORC1's inhibitory subunit AKT1S1, leading to mTORC1 activation (Wippich et al., 2013). In response to stress SGs recruit inactive DYRK3, allowing active AKT1S1 to suppress mTORC1 (Wippich et al., 2013; Mediani et al., 2021). DYRK3 stabilizes SGs, enhancing inhibitory effects of SGs on mTORC1. Next to the inhibitory cues, activating stress inputs (Wang and Proud, 1997; White et al., 2007; 
Wu et al., 2011; Sfakianos et al., 2018; Heberle et al., 2019) finely orchestrate mTORC1 activity. mTORC1 enhances SG formation by several mechanisms that involve mediators of translation and autophagy (Fournier et al., 2013; Mazan-Mamczarz et al., 2015; Sfakianos et al., 2018; Zhang et al., 2018). Via such SG-mediated negative feedback mTORC1 may restrict its own activity under stress.

\section{Crosstalk of Lysosomes and SGs in TSC Complex-mTORC1 Signaling}

Several findings indicate crosstalk between lysosomes and SGs. Absence of SPAG5 not only reduces SG tethering of RPTOR but also enhances its binding to lysosomes (Thedieck et al., 2013). In agreement, in the absence of SGs, the core SG proteins and bona fide markers of SG assembly G3BP1 and 2 (Riggs et al., 2020) reside at the cytoplasmic surface of lysosomes and function as tethers of the TSC complex (Prentzell et al., 2021; Figure 1). The C-terminal RGG (arginine-glycineglycine) domain of G3BP1 binds to TSC2 and the N-terminal NTF2L domain of G3BP1 binds to the lysosomal associated membrane proteins $1 / 2$ (LAMP1/2), bridging the TSC complex to the lysosomal surface (Prentzell et al., 2021). G3BPs suppress mTORC1 signaling in the presence as well as in the absence of nutrients (growth factors and amino acids). In keeping with a function in lysosomal tethering of the TSC complex, G3BP1 inhibition is sufficient to phenocopy loss of TSC2 with regard to (i) mTORC1 hyperactivity, (ii) increased cell size, and (iii) enhanced lysosomal MTOR localization (Prentzell et al., 2021). Next to G3BPs, also the RHEB and RRAG GTPases contribute to the lysosomal recruitment of the TSC complex (Demetriades et al., 2014; Menon et al., 2014; Carroll et al., 2016; Yang et al., 2020). G3BP1 and RHEB deficiency reduce lysosomal TSC2 localization to a similar extent, without additive effects (Prentzell et al., 2021), indicating that both mechanisms are required for efficient lysosomal recruitment of the TSC complex. Of note, G3BP1 deficiency does not activate mTORC1 signaling in the presence of SGs (Prentzell et al., 2021) suggesting that the G3BPs' functions at lysosomes and in SGs are mutually exclusive. It is tempting to speculate that in response to stress G3BP proteins shuttle from the lysosomes to SGs. Thus, stress may reduce lysosomal tethering of the TSC complex by G3BP to sustain mTORC1 activity. However, the TSC complex suppresses mTORC1 also under stress (Plescher et al., 2015; Demetriades et al., 2016) and mechanisms other than G3BP-TSC2 may take over for the lysosomal tethering of the TSC complex. Findings of Fitzian et al. (2021) suggest the involvement of lysosomal phospholipids as TSC1 binds PI(3,5)P2 (phosphatidylinositol-3,5-bisphosphate) in a charge dependent manner (Figure 1). Osmotic stress enhances PI(3,5)P2 levels in the lysosomal membrane (Jin et al., 2017), and it is conceivable that lysosomal tethering of the TSC complex via TSC1 becomes dominant under stress conditions. Future studies on the cooperation between different modes of lysosomal TSC complex tethering will reveal which mechanisms dominate upon different metabolic and stress stimuli.
Whereas G3BPs tether the TSC complex to lysosomes under nutrient sufficiency, oxidative (i.e., sodium arsenite) and heat stress induce the recruitment of TSC2 to G3BP1-positive SGs (Kosmas et al., 2021). SG recruitment of TSC2 is mediated by its interaction with high density lipoprotein binding protein (HDLBP, also named Vigilin), whose SG localization was discovered first in yeast (Wen et al., 2010). HDLBP appeared in two omics-wide analyses of SGs (Markmiller et al., 2018; Youn et al., 2018) and was shown recently to localize to SGs also in mammalian cells (Kosmas et al., 2021; Figure 1). Knockdown of HDLBP reduces TSC2 localization to SGs while not affecting SG formation, indicating that HDLBP mediates the SG recruitment of TSC2 (Kosmas et al., 2021). Interestingly, TSC2 deficiency enhances the number of G3BP1-positive SGs. In agreement, mTORC1 activity promotes SG assembly (Fournier et al., 2013; Sfakianos et al., 2018; Heberle et al., 2019), possibly constituting the mechanism via which TSC2 deficiency enhances SG assembly. It will be interesting to investigate whether stressinduced TSC2 translocation from the lysosomes to SGs elicits a positive feedback loop. By de-repression of mTORC1 at lysosomes, such positive feedback may enhance the formation of SGs and SG recruitment of TSC2. Intriguingly, under conditions of nutrient sufficiency (i.e., in the absence of SGs) not only the SG proteins G3BP1 and 2 (Prentzell et al., 2021), but also HDLBP (Wyant et al., 2018) reside at the lysosomes. HDLBP's lysosomal function is still unknown and it remains open whether it also acts on lysosomal TSC2.

To conclude, G3BPs, SPAG5, and possibly HDLBP have dual roles at lysosomes and SGs (Thedieck et al., 2013; Kosmas et al., 2021; Prentzell et al., 2021). Of note, Liao et al. (2019) showed that ANXA11 (Annexin A11) tethers SGs to lysosomes for distal traveling in neurons. It is conceivable that such close proximity allows proteins to shuttle between lysosomes and SGs. Future research will tackle this question and may reveal the underlying mechanisms. The proximity of lysosomes and SGs may also explain observations that autophagy, one of the major functions of the lysosomal compartment, mediates SG clearance (Buchan et al., 2013; Marrone et al., 2018; Zhang et al., 2018; Silva et al., 2019) and their proper assembly (Seguin et al., 2014). mTORC1 is one of the key suppressors of autophagy as it inhibits autophagosome initiation by ULK1 and ATG13 (autophagy related 13) (Deleyto-Seldas and Efeyan, 2021). mTORC1 also inhibits TFEB and TFE3 (transcription factor $\mathrm{EB}$, transcription factor binding to IGHM enhancer 3), major transcription factors of the autophagic-lysosomal pathway [reviewed by Noda et al. (2020)]. The involvement of SG proteins in lysosomal mTORC1 suppression may link them to autophagy and the turnover and assembly of the SG compartment.

\section{TSC Complex Tethers at Lysosomes and SGs in Human Disease}

G3BP1 promotes proliferation of breast cancer cells (Winslow et al., 2013; Prentzell et al., 2021; Zhang et al., 2021) and in a TSC2-deficient tumor model (Kosmas et al., 2021). G3BP1 mRNA levels are increased in mouse and human TSC tumors 
[angiomyolipomas (AML), subependymal giant cell astrocytoma (SEGA), subependymal nodules (SEN)] (Kosmas et al., 2021) and in breast cancer (Winslow et al., 2013; Zhang et al., 2021). This suggests that G3BPs may be targets for tumor treatment. In keeping with this, inhibition of G3BP1 enhances apoptosis in TSC2-deficient cells in vitro (Kosmas et al., 2021). G3BP1 inhibition also prolongs tumor-free survival and represses tumor growth in a subcutaneous in vivo model derived from a TSC2deficient renal tumor (Kosmas et al., 2021). This may have implications for the many proliferative lesions in TSC, which include renal AML, cardiac rhabdomyomas, and SEGAs (Henske et al., 2016). However, G3BP1 also suppresses migration in an mTORC1-dependent manner (Prentzell et al., 2021; Figure 1), suggesting that targeting G3BP1 may be contraindicated in some situations, such as breast cancer, in which lower levels of G3BP1, TSC1, and TSC2 are associated with reduced relapsefree survival (Prentzell et al., 2021). It is unknown whether G3BP1 controls TSC-associated tumors in human patients. G3BP1-dependent cell migration may be particularly important for women with LAM, the pulmonary manifestation of TSC, in which TSC2-deficient smooth muscle-like cells migrate to the lungs and cause emphysema-like lung destruction (Henske and McCormack, 2012). G3BP1-dependent migration may be also of clinical importance for cerebral cortical tubers in TSC, which are believed to arise from aberrant neuronal migration (Henske et al., 2016). The seemingly contradictory findings on G3BPs in tumors may result from G3BP1's dual roles at SGs and lysosomes, respectively. Whereas SGs suppress cell death, making G3BP1 pro-tumorigenic, mTORC1 inhibition at lysosomes rather highlights the G3BPs as tumor suppressors. G3BPs also have a role in other oncogenic pathways, including RAS (Parker et al., 1996), NFKB1 (nuclear factor kappa B subunit 1) (Prigent et al., 2000), WNT (Bikkavilli and Malbon, 2011), and TGFB (Zhang et al., 2015). The G3BPs' function that dominates in a given tumor may determine whether an intervention at the level of the G3BPs is pro- or anti-tumorigenic.

On a broader level, the new data on lysosomal and SG tethers of the TSC complex may impact our understanding of the pathogenesis and therapy of the many diseases in which dysregulation of the TSC complex-mTORC1 axis is observed. Diseases in which mTORC1 has a key role include the majority of human malignancies (Hoxhaj and Manning, 2020), as well as diabetes, obesity, and aging (Papadopoli et al., 2019; Liu and Sabatini, 2020). Understanding how the functions of G3BPs, $\mathrm{PI}(3,5) \mathrm{P} 2$ and HDLBP in TSC subunit recruitment to lysosomes are integrated into the pathobiology of these diseases could have wide-ranging implications for human health. Like the G3BPs, also HDLBP (Yang et al., 2014) and PI(3,5)P2 (Hou et al., 2019; Ikonomov et al., 2019) control proliferation and migration of cancer cells. In agreement, altered HDLBP levels have been reported in different tumor entities (Yang et al., 2014; Woo et al., 2019), and PI(3,5)P2 and the G3BPs have been linked to malignancies and neuronal disorders (Wallroth and Haucke, 2018; Mandal, 2020; Prentzell et al., 2021). These disorders may arise, at least in part, from aberrant lysosomal TSC complex levels and mTORC1 activity.

\section{OUTLOOK}

To conclude, several mechanisms tether the TSC complex to lysosomes as well as to SGs, and control its inhibitory function toward mTORC1. Future research will unravel cooperation and competition between RHEB, RRAGs, G3BP1/2, PI(3,5)P2 and HDLBP in tethering the TSC complex to lysosomes and SGs and in controlling proliferation and migration under different metabolic and stress conditions. This may be clinically relevant for diseases characterized by dysregulated TSC complex and mTORC1 activity. G3BP proteins have been proposed as therapeutic targets based on their role in SG assembly (Zhang et al., 2012, 2019; Alam and Kennedy, 2019; Anisimov et al., 2019; Kosmas et al., 2021). However, their lysosomal TSC complextethering function warrants cautious evaluation of this concept in a tumor- and context-specific manner as G3BPs suppress oncogenic mTORC1 signaling. As HDLBP resides not only at SGs but also at lysosomes, it may give rise to pleiotropic effects similar to G3BPs that are to be investigated in future studies. G3BPs and HDLBP may represent indicators of mTORC1 activity with utility as predictive biomarkers for the response to drugs targeting mTORC1. Such applications will require careful investigation in clinical trials with inhibitors of mTORC1 and its upstream kinases.

\section{AUTHOR CONTRIBUTIONS}

UR and KT wrote the first draft of the manuscript. UR, MTP, AH, MCS, $\mathrm{EH}, \mathrm{CO}$, and $\mathrm{KT}$ contributed to the manuscript writing, read, and approved the final version. All authors contributed to the article and approved the submitted version.

\section{FUNDING}

We acknowledge support from research awards from the German Tuberous Sclerosis Foundation 2019 (to MTP) and 2017 (to KT); the German Research Foundation (SFB 430 1389-UNITE Glioblastoma to CO); the German TS Foundation (to KT); Stichting TSC Fonds (to KT); the PoLiMeR Innovative Training Network (Marie Skłodowska-Curie grant agreement 812616 to KT); and the MESI-STRAT project (grant agreement 754688 to $\mathrm{CO}$ and $\mathrm{KT}$ ), which received funding from the European Union Horizon 2020 Research and Innovation Program. MCS acknowledges support from the Graduate School of Medical Sciences of the University of Groningen. MCS and UR acknowledge support from the Vice Rectorate for Research of the University of Innsbruck in the course of Early Stage Funding 2021.

\section{ACKNOWLEDGMENTS}

The figure was created with BioRender.com (Toronto, ON, Canada). The protein names follow the HGNC (HUGO Gene Nomenclature Committee) standard. 


\section{REFERENCES}

Advani, V. M., and Ivanov, P. (2019). Translational control under stress: reshaping the translatome. Bioessays 41:e1900009. doi: 10.1002/bies.201900009

Alam, U., and Kennedy, D. (2019). Rasputin a decade on and more promiscuous than ever? A review of G3BPs. Biochim. Biophys. Acta Mol. Cell Res. 1866, 360-370. doi: 10.1016/j.bbamcr.2018.09.001

Alberti, S., and Dormann, D. (2019). Liquid-liquid phase separation in disease. Annu. Rev. Genet. 53, 171-194. doi: 10.1146/annurev-genet-112618-043527

Anderson, P., Kedersha, N., and Ivanov, P. (2015). Stress granules, P-bodies and cancer. Biochim. Biophys. Acta 1849, 861-870. doi: 10.1016/j.bbagrm.2014.11. 009

Anisimov, S., Takahashi, M., Kakihana, T., Katsuragi, Y., Kitaura, H., Zhang, L., et al. (2019). G3BP1 inhibits ubiquitinated protein aggregations induced by p62 and USP10. Sci. Rep. 9:12896. doi: 10.1038/s41598-019-46237-1

Arimoto, K., Fukuda, H., Imajoh-Ohmi, S., Saito, H., and Takekawa, M. (2008). Formation of stress granules inhibits apoptosis by suppressing stress-responsive MAPK pathways. Nat. Cell Biol. 10, 1324-1332. doi: 10.1038/ncb1791

Bikkavilli, R. K., and Malbon, C. C. (2011). Arginine methylation of G3BP1 in response to Wnt3a regulates beta-catenin mRNA. J. Cell Sci. 124(Pt 13), 2310-2320. doi: 10.1242/jcs.084046

Borkowska, J., Schwartz, R. A., Kotulska, K., and Jozwiak, S. (2011). Tuberous sclerosis complex: tumors and tumorigenesis. Int. J. Dermatol. 50, 13-20. doi: 10.1111/j.1365-4632.2010.04727.x

Buchan, J. R., Kolaitis, R. M., Taylor, J. P., and Parker, R. (2013). Eukaryotic stress granules are cleared by autophagy and Cdc48/VCP function. Cell 153, 1461-1474. doi: 10.1016/j.cell.2013.05.037

Carroll, B., Maetzel, D., Maddocks, O. D., Otten, G., Ratcliff, M., Smith, G. R., et al. (2016). Control of TSC2-Rheb signaling axis by arginine regulates mTORC1 activity. eLife 5:e11058. doi: 10.7554/eLife.11058

Chao, L. H., and Avruch, J. (2019). Cryo-EM insight into the structure of MTOR complex 1 and its interactions with Rheb and substrates. F1000Res. 8:F1000 Faculty Rev-14. doi: 10.12688/f1000research.16109.1

Condon, K. J., and Sabatini, D. M. (2019). Nutrient regulation of mTORC1 at a glance. J. Cell Sci. 132:jcs222570. doi: 10.1242/jcs.222570

Curatolo, P., Moavero, R., and de Vries, P. J. (2015). Neurological and neuropsychiatric aspects of tuberous sclerosis complex. Lancet Neurol. 14, 733-745. doi: 10.1016/S1474-4422(15)00069-1

Deleyto-Seldas, N., and Efeyan, A. (2021). The mTOR-autophagy axis and the control of metabolism. Front. Cell Dev. Biol. 9:655731. doi: 10.3389/fcell.2021. 655731

Demetriades, C., Doumpas, N., and Teleman, A. A. (2014). Regulation of TORC1 in response to amino acid starvation via lysosomal recruitment of TSC2. Cell 156, 786-799. doi: 10.1016/j.cell.2014.01.024

Demetriades, C., Plescher, M., and Teleman, A. A. (2016). Lysosomal recruitment of TSC2 is a universal response to cellular stress. Nat. Commun. 7:10662. doi: $10.1038 /$ ncomms 10662

Dibble, C. C., Elis, W., Menon, S., Qin, W., Klekota, J., Asara, J. M., et al. (2012). TBC1D7 is a third subunit of the TSC1-TSC2 complex upstream of mTORC1. Mol. Cell 47, 535-546. doi: 10.1016/j.molcel.2012.06.009

Fernandes, S. A., and Demetriades, C. (2021). The multifaceted role of nutrient sensing and mTORC1 signaling in physiology and aging. Front. Aging 2:707372. doi: 10.3389/fragi.2021.707372

Fitzian, K., Bruckner, A., Brohee, L., Zech, R., Antoni, C., Kiontke, S., et al. (2021). TSC1 binding to lysosomal PIPs is required for TSC complex translocation and mTORC1 regulation. Mol. Cell 81, 2705.e8-2721.e8. doi: 10.1016/j.molcel.2021. 04.019

Fournier, M. J., Coudert, L., Mellaoui, S., Adjibade, P., Gareau, C., Cote, M. F., et al. (2013). Inactivation of the mTORC1-eukaryotic translation initiation factor 4E pathway alters stress granule formation. Mol. Cell Biol. 33, 2285-2301. doi: 10.1128/MCB.01517-12

Gonzalez, A., Hall, M. N., Lin, S. C., and Hardie, D. G. (2020). AMPK and TOR: the yin and yang of cellular nutrient sensing and growth control. Cell Metab. 31, 472-492. doi: 10.1016/j.cmet.2020.01.015

Heberle, A. M., Prentzell, M. T., van Eunen, K., Bakker, B. M., Grellscheid, S. N., and Thedieck, K. (2015). Molecular mechanisms of mTOR regulation by stress. Mol. Cell Oncol. 2:e970489. doi: 10.4161/23723548.2014.970489
Heberle, A. M., Razquin Navas, P., Langelaar-Makkinje, M., Kasack, K., Sadik, A., Faessler, E., et al. (2019). The PI3K and MAPK/p38 pathways control stress granule assembly in a hierarchical manner. Life Sci. Alliance 2:e201800257. doi: 10.26508/lsa.201800257

Henske, E. P., Jozwiak, S., Kingswood, J. C., Sampson, J. R., and Thiele, E. A. (2016). Tuberous sclerosis complex. Nat. Rev. Dis. Primers 2:16035. doi: 10.1038/nrdp. 2016.35

Henske, E. P., and McCormack, F. X. (2012). Lymphangioleiomyomatosis - a wolf in sheep's clothing. J. Clin. Invest. 122, 3807-3816. doi: 10.1172/JCI58709

Hofmann, S., Kedersha, N., Anderson, P., and Ivanov, P. (2021). Molecular mechanisms of stress granule assembly and disassembly. Biochim. Biophys. Acta Mol. Cell Res. 1868:118876. doi: 10.1016/j.bbamcr.2020.118876

Holcik, M. (2015). Could the eIF2alpha-independent translation be the Achilles heel of cancer? Front. Oncol. 5:264. doi: 10.3389/fonc.2015.00264

Hou, J. Z., Xi, Z. Q., Niu, J., Li, W., Wang, X., Liang, C., et al. (2019). Inhibition of PIKfyve using YM201636 suppresses the growth of liver cancer via the induction of autophagy. Oncol. Rep. 41, 1971-1979. doi: 10.3892/or.2018. 6928

Hoxhaj, G., and Manning, B. D. (2020). The PI3K-AKT network at the interface of oncogenic signalling and cancer metabolism. Nat. Rev. Cancer 20, 74-88. doi: 10.1038/s41568-019-0216-7

Huang, J., and Manning, B. D. (2008). The TSC1-TSC2 complex: a molecular switchboard controlling cell growth. Biochem. J. 412, 179-190. doi: 10.1042/ BJ20080281

Ikonomov, O. C., Sbrissa, D., and Shisheva, A. (2019). Small molecule PIKfyve inhibitors as cancer therapeutics: translational promises and limitations. Toxicol Appl. Pharmacol. 383:114771. doi: 10.1016/j.taap.2019.114771

Inoki, K., Zhu, T., and Guan, K. L. (2003b). TSC2 mediates cellular energy response to control cell growth and survival. Cell 115, 577-590. doi: 10.1016/s00928674(03)00929-2

Inoki, K., Li, Y., Xu, T., and Guan, K. L. (2003a). Rheb GTPase is a direct target of TSC2 GAP activity and regulates mTOR signaling. Genes Dev. 17, 1829-1834. doi: $10.1101 / \operatorname{gad} .1110003$

Inoki, K., Li, Y., Zhu, T., Wu, J., and Guan, K. L. (2002). TSC2 is phosphorylated and inhibited by Akt and suppresses mTOR signalling. Nat. Cell Biol. 4, 648-657. doi: 10.1038/ncb839

Inoki, K., Ouyang, H., Zhu, T., Lindvall, C., Wang, Y., Zhang, X., et al. (2006). TSC2 integrates Wnt and energy signals via a coordinated phosphorylation by AMPK and GSK3 to regulate cell growth. Cell 126, 955-968. doi: 10.1016/j.cell.2006.0 6.055

Jin, N., Jin, Y., and Weisman, L. S. (2017). Early protection to stress mediated by CDK-dependent PI3,5P2 signaling from the vacuole/lysosome. J. Cell Biol. 216, 2075-2090. doi: 10.1083/jcb.201611144

Jozwiak, S., Kotulska, K., Wong, M., and Bebin, M. (2019). Modifying genetic epilepsies - Results from studies on tuberous sclerosis complex. Neuropharmacology 166:107908. doi: 10.1016/j.neuropharm.2019.107908

Kedersha, N., Ivanov, P., and Anderson, P. (2013). Stress granules and cell signaling: more than just a passing phase? Trends Biochem. Sci. 38, 494-506. doi: 10.1016/j.tibs.2013.07.004

Kim, J., and Guan, K. L. (2019). mTOR as a central hub of nutrient signalling and cell growth. Nat. Cell Biol. 21, 63-71. doi: 10.1038/s41556-018-0205-1

Kim, W. J., Back, S. H., Kim, V., Ryu, I., and Jang, S. K. (2005). Sequestration of TRAF2 into stress granules interrupts tumor necrosis factor signaling under stress conditions. Mol. Cell Biol. 25, 2450-2462. doi: 10.1128/MCB.25.6.24502462.2005

Kosmas, K., Filippakis, H., Khabibullin, D., Turkiewicz, M., Lam, H. C., Yu, J., et al. (2021). TSC2 Interacts with HDLBP/Vigilin and regulates stress granule formation. Mol. Cancer Res. 19, 1389-1397. doi: 10.1158/1541-7786.MCR-201046

Lastres-Becker, I., Nonis, D., Eich, F., Klinkenberg, M., Gorospe, M., Kotter, P., et al. (2016). Mammalian ataxin-2 modulates translation control at the preinitiation complex via PI3K/mTOR and is induced by starvation. Biochim. Biophys. Acta 1862, 1558-1569. doi: 10.1016/j.bbadis.2016.05.017

Liao, Y. C., Fernandopulle, M. S., Wang, G., Choi, H., Hao, L., Drerup, C. M., et al. (2019). RNA granules hitchhike on lysosomes for long-distance transport, using annexin A11 as a molecular tether. Cell 179, 147.e20-164.e20. doi: 10.1016/j.cell. 2019.08.050 
Liu, G. Y., and Sabatini, D. M. (2020). mTOR at the nexus of nutrition, growth, ageing and disease. Nat. Rev. Mol. Cell Biol. 21, 183-203. doi: 10.1038/s41580019-0199-y

Ma, L., Chen, Z., Erdjument-Bromage, H., Tempst, P., and Pandolfi, P. P. (2005). Phosphorylation and functional inactivation of TSC2 by Erk implications for tuberous sclerosis and cancer pathogenesis. Cell 121, 179-193. doi: 10.1016/j. cell.2005.02.031

Mandal, K. (2020). Review of PIP2 in cellular signaling, functions and diseases. Int. J. Mol. Sci. 21:8342. doi: 10.3390/ijms21218342

Manning, B. D., Tee, A. R., Logsdon, M. N., Blenis, J., and Cantley, L. C. (2002). Identification of the tuberous sclerosis complex-2 tumor suppressor gene product tuberin as a target of the phosphoinositide 3-kinase/akt pathway. Mol. Cell 10, 151-162. doi: 10.1016/s1097-2765(02)00568-3

Markmiller, S., Soltanieh, S., Server, K. L., Mak, R., Jin, W., Fang, M. Y., et al. (2018). Context-dependent and disease-specific diversity in protein interactions within stress granules. Cell 172, 590.e13-604.e13. doi: 10.1016/j.cell.2017.1 2.032

Marrone, L., Poser, I., Casci, I., Japtok, J., Reinhardt, P., Janosch, A., et al. (2018). Isogenic FUS-eGFP iPSC reporter lines enable quantification of FUS stress granule pathology that is rescued by drugs inducing autophagy. Stem Cell Rep. 10, 375-389. doi: 10.1016/j.stemcr.2017.12.018

Mazan-Mamczarz, K., Peroutka, R. J., Steinhardt, J. J., Gidoni, M., Zhang, Y., Lehrmann, E., et al. (2015). Distinct inhibitory effects on mTOR signaling by ethanol and INK128 in diffuse large B-cell lymphoma. Cell Commun. Signal. 13:15. doi: 10.1186/s12964-015-0091-0

Mediani, L., Antoniani, F., Galli, V., Vinet, J., Carra, A. D., Bigi, I., et al. (2021). Hsp90-mediated regulation of DYRK3 couples stress granule disassembly and growth via mTORC1 signaling. EMBO Rep. 22:e51740. doi: 10.15252/embr. 202051740

Menon, S., Dibble, C. C., Talbott, G., Hoxhaj, G., Valvezan, A. J., Takahashi, H., et al. (2014). Spatial control of the TSC complex integrates insulin and nutrient regulation of mTORC1 at the lysosome. Cell 156, 771-785. doi: 10.1016/j.cell. 2013.11.049

Mossmann, D., Park, S., and Hall, M. N. (2018). mTOR signalling and cellular metabolism are mutual determinants in cancer. Nat. Rev. Cancer 18, 744-757. doi: $10.1038 /$ s41568-018-0074-8

Noda, N. N., Wang, Z., and Zhang, H. (2020). Liquid-liquid phase separation in autophagy. J. Cell Biol. 219:e202004062. doi: 10.1083/jcb.202004062

Oakhill, J. S., Chen, Z. P., Scott, J. W., Steel, R., Castelli, L. A., Ling, N., et al. (2010). $\beta$-Subunit myristoylation is the gatekeeper for initiating metabolic stress sensing by AMP-activated protein kinase (AMPK). Proc. Natl. Acad. Sci. U.S.A. 107, 19237-19241. doi: 10.1073/pnas.1009705107

Orlova, K. A., and Crino, P. B. (2010). The tuberous sclerosis complex. Ann. N. Y. Acad. Sci. 1184, 87-105. doi: 10.1111/j.1749-6632.2009.05117.x

Papadopoli, D., Boulay, K., Kazak, L., Pollak, M., Mallette, F., Topisirovic, I., et al. (2019). mTOR as a central regulator of lifespan and aging. F1000Res. 8:F1000 Faculty Rev-998. doi: 10.12688/f1000research.17196.1

Park, Y. J., Choi, D. W., Cho, S. W., Han, J., Yang, S., and Choi, C. Y. (2020). Stress granule formation attenuates RACK1-mediated apoptotic cell death induced by morusin. Int. J. Mol. Sci. 21:5360. doi: 10.3390/ijms21155360

Panas, M. D., Ivanov, P., and Anderson, P. (2016). Mechanistic insights into mammalian stress granule dynamics. J. Cell Biol. 215, 313-323. doi: 10.1083/ jcb.201609081

Parker, F., Maurier, F., Delumeau, I., Duchesne, M., Faucher, D., Debussche, L., et al. (1996). A Ras-GTPase-activating protein SH3-domain-binding protein. Mol. Cell Biol. 16, 2561-2569.

Pla-Martin, D., Schatton, D., Wiederstein, J. L., Marx, M. C., Khiati, S., Kruger, M., et al. (2020). CLUH granules coordinate translation of mitochondrial proteins with mTORC1 signaling and mitophagy. EMBO J. 39:e102731. doi: 10.15252/ embj.2019102731

Plescher, M., Teleman, A. A., and Demetriades, C. (2015). TSC2 mediates hyperosmotic stress-induced inactivation of mTORC1. Sci. Rep. 5:13828. doi: 10.1038/srep 13828

Potter, C. J., Pedraza, L. G., and Xu, T. (2002). Akt regulates growth by directly phosphorylating Tsc2. Nat. Cell Biol. 4, 658-665. doi: 10.1038/ncb840

Prentzell, M. T., Rehbein, U., Cadena Sandoval, M., De Meulemeester, A. S., Baumeister, R., Brohee, L., et al. (2021). G3BPs tether the TSC complex to lysosomes and suppress mTORC1 signaling. Cell 184, 655.e27-674.e27. doi: 10.1016/j.cell.2020.12.024
Prigent, M., Barlat, I., Langen, H., and Dargemont, C. (2000). IkappaBalpha and IkappaBalpha/NF-kappa B complexes are retained in the cytoplasm through interaction with a novel partner. RasGAP SH3-binding protein 2. J. Biol. Chem. 275, 36441-36449. doi: 10.1074/jbc.M004751200

Rabanal-Ruiz, Y., and Korolchuk, V. I. (2018). mTORC1 and nutrient homeostasis: the central role of the lysosome. Int. J. Mol. Sci. 19:818. doi: 10.3390/ ijms 19030818

Ramiscal, R. R., Parish, I. A., Lee-Young, R. S., Babon, J. J., Blagih, J., Pratama, A., et al. (2015). Attenuation of AMPK signaling by ROQUIN promotes $\mathrm{T}$ follicular helper cell formation. eLife 4:e08698. doi: 10.7554/eLife.08698

Ramlaul, K., Fu, W., Li, H., de Martin Garrido, N., He, L., Trivedi, M., et al. (2021). Architecture of the tuberous sclerosis protein complex. J. Mol. Biol. 433:166743. doi: 10.1016/j.jmb.2020.166743

Riggs, C. L., Kedersha, N., Ivanov, P., and Anderson, P. (2020). Mammalian stress granules and P bodies at a glance. J. Cell Sci. 133:jcs242487. doi: 10.1242/jcs. 242487

Roux, P. P., Ballif, B. A., Anjum, R., Gygi, S. P., and Blenis, J. (2004). Tumorpromoting phorbol esters and activated Ras inactivate the tuberous sclerosis tumor suppressor complex via p90 ribosomal S6 kinase. Proc. Natl. Acad. Sci. U.S.A. 101, 13489-13494. doi: 10.1073/pnas.0405659101

Seguin, S. J., Morelli, F. F., Vinet, J., Amore, D., De Biasi, S., Poletti, A., et al. (2014). Inhibition of autophagy, lysosome and VCP function impairs stress granule assembly. Cell Death Differ. 21, 1838-1851. doi: 10.1038/cdd.2014.103

Sfakianos, A. P., Mellor, L. E., Pang, Y. F., Kritsiligkou, P., Needs, H., AbouHamdan, H., et al. (2018). The mTOR-S6 kinase pathway promotes stress granule assembly. Cell Death Differ. 25, 1766-1780. doi: 10.1038/s41418-0180076-9

Silva, J. M., Rodrigues, S., Sampaio-Marques, B., Gomes, P., Neves-Carvalho, A., Dioli, C., et al. (2019). Dysregulation of autophagy and stress granule-related proteins in stress-driven Tau pathology. Cell Death Differ. 26, 1411-1427. doi: 10.1038/s41418-018-0217-1

Takahara, T., and Maeda, T. (2012). Transient sequestration of TORC1 into stress granules during heat stress. Mol. Cell 47, 242-252. doi: 10.1016/j.molcel.2012. 05.019

Tee, A. R. (2018). The target of rapamycin and mechanisms of cell growth. Int. J. Mol. Sci. 19:880. doi: 10.3390/ijms19030880

Tee, A. R., Manning, B. D., Roux, P. P., Cantley, L. C., and Blenis, J. (2003). Tuberous sclerosis complex gene products, Tuberin and Hamartin, control mTOR signaling by acting as a GTPase-activating protein complex toward Rheb. Curr. Biol. 13, 1259-1268. doi: 10.1016/s0960-9822(03)00506-2

Thedieck, K., Holzwarth, B., Prentzell, M. T., Boehlke, C., Klasener, K., Ruf, S., et al. (2013). Inhibition of mTORC1 by astrin and stress granules prevents apoptosis in cancer cells. Cell 154, 859-874. doi: 10.1016/j.cell.2013.07.031

Thien, A., Prentzell, M. T., Holzwarth, B., Klasener, K., Kuper, I., Boehlke, C., et al. (2015). TSC1 activates TGF-beta-Smad2/3 signaling in growth arrest and epithelial-to-mesenchymal transition. Dev. Cell 32, 617-630. doi: 10.1016/j. devcel.2015.01.026

Tsai, N. P., and Wei, L. N. (2010). RhoA/ROCK1 signaling regulates stress granule formation and apoptosis. Cell Signal 22, 668-675. doi: 10.1016/j.cellsig.2009.1 2.001

Wallroth, A., and Haucke, V. (2018). Phosphoinositide conversion in endocytosis and the endolysosomal system. J. Biol. Chem. 293, 1526-1535. doi: 10.1074/jbc. R117.000629

Wang, X., and Proud, C. G. (1997). p70 S6 kinase is activated by sodium arsenite in adult rat cardiomyocytes: roles for phosphatidylinositol 3-kinase and p38 MAP kinase. Biochem. Biophys. Res. Commun. 238, 207-212. doi: 10.1006/bbrc.1997. 7273

Wen, W. L., Stevenson, A. L., Wang, C. Y., Chen, H. J., Kearsey, S. E., Norbury, C. J., et al. (2010). Vgl1, a multi-KH domain protein, is a novel component of the fission yeast stress granules required for cell survival under thermal stress. Nucleic Acids Res. 38, 6555-6566. doi: 10.1093/nar/gkq555

White, J. P., Cardenas, A. M., Marissen, W. E., and Lloyd, R. E. (2007). Inhibition of cytoplasmic mRNA stress granule formation by a viral proteinase. Cell Host Microbe 2, 295-305. doi: 10.1016/j.chom.2007.08.006

Winslow, S., Leandersson, K., and Larsson, C. (2013). Regulation of PMP22 mRNA by G3BP1 affects cell proliferation in breast cancer cells. Mol. Cancer 12:156. doi: 10.1186/1476-4598-12-156

Wippich, F., Bodenmiller, B., Trajkovska, M. G., Wanka, S., Aebersold, R., and Pelkmans, L. (2013). Dual specificity kinase DYRK3 couples stress granule 
condensation/dissolution to mTORC1 signaling. Cell 152, 791-805. doi: 10 . 1016/j.cell.2013.01.033

Woo, H. H., Lee, S. C., Stoffer, J. B., Rush, D., and Chambers, S. K. (2019). Phenotype of vigilin expressing breast cancer cells binding to the $69 \mathrm{nt} 3^{\prime} \mathrm{UTR}$ element in CSF-1R mRNA. Transl. Oncol. 12, 106-115. doi: 10.1016/j.tranon. 2018.09.012

Wu, X. N., Wang, X. K., Wu, S. Q., Lu, J., Zheng, M., Wang, Y. H., et al. (2011). Phosphorylation of Raptor by p38beta participates in arsenite-induced mammalian target of rapamycin complex 1 (mTORC1) activation. J. Biol. Chem. 286, 31501-31511. doi: 10.1074/jbc.M111.233122

Wyant, G. A., Abu-Remaileh, M., Frenkel, E. M., Laqtom, N. N., Dharamdasani, V., Lewis, C. A., et al. (2018). NUFIP1 is a ribosome receptor for starvation-induced ribophagy. Science 360, 751-758. doi: 10.1126/science.aar2663

Yang, H., Jiang, X., Li, B., Yang, H. J., Miller, M., Yang, A., et al. (2017). Mechanisms of mTORC1 activation by RHEB and inhibition by PRAS40. Nature 552, 368-373. doi: 10.1038/nature25023

Yang, H., Rudge, D. G., Koos, J. D., Vaidialingam, B., Yang, H. J., and Pavletich, N. P. (2013). mTOR kinase structure, mechanism and regulation. Nature 497, 217-223. doi: 10.1038/nature12122

Yang, H., Yu, Z., Chen, X., Li, J., Li, N., Cheng, J., et al. (2021). Structural insights into TSC complex assembly and GAP activity on Rheb. Nat. Commun. 12:339. doi: 10.1038/s41467-020-20522-4

Yang, S., Zhang, Y., Ting, C. Y., Bettedi, L., Kim, K., Ghaniam, E., et al. (2020). The Rag GTPase regulates the dynamic behavior of tsc downstream of both amino acid and growth factor restriction. Dev. Cell 55, 272.e5-288.e5. doi: 10.1016/j.devcel.2020.08.006

Yang, W. L., Wei, L., Huang, W. Q., Li, R., Shen, W. Y., Liu, J. Y., et al. (2014). Vigilin is overexpressed in hepatocellular carcinoma and is required for HCC cell proliferation and tumor growth. Oncol. Rep. 31, 2328-2334. doi: 10.3892/ or.2014.3111

Yip, C. K., Murata, K., Walz, T., Sabatini, D. M., and Kang, S. A. (2010). Structure of the human mTOR complex I and its implications for rapamycin inhibition. Mol. Cell 38, 768-774. doi: 10.1016/j.molcel.2010.05.017

Youn, J. Y., Dunham, W. H., Hong, S. J., Knight, J. D. R., Bashkurov, M., Chen, G. I., et al. (2018). High-density proximity mapping reveals the subcellular organization of mRNA-associated granules and bodies. Mol. Cell 69, 517.e11532.e11. doi: 10.1016/j.molcel.2017.12.020

Zhang, G., Wang, Z., Du, Z., and Zhang, H. (2018). mTOR regulates phase separation of PGL granules to modulate their autophagic degradation. Cell 174, 1492.e22-1506.e22. doi: 10.1016/j.cell.2018. 08.006

Zhang, H., Ma, Y., Zhang, S., Liu, H., He, H., Li, N., et al. (2015). Involvement of Ras GTPase-activating protein $\mathrm{SH} 3$ domain-binding protein 1 in the epithelialto-mesenchymal transition-induced metastasis of breast cancer cells via the Smad signaling pathway. Oncotarget 6, 17039-17053. doi: 10.18632/oncotarget. 3636

Zhang, H., Zhang, S., He, H., Zhao, W., Chen, J., and Shao, R. G. (2012). GAP161 targets and downregulates G3BP to suppress cell growth and potentiate cisplaitin-mediated cytotoxicity to colon carcinoma HCT116 cells. Cancer Sci. 103, 1848-1856. doi: 10.1111/j.1349-7006.2012.02361.x

Zhang, P., Fan, B., Yang, P., Temirov, J., Messing, J., Kim, H. J., et al. (2019). Chronic optogenetic induction of stress granules is cytotoxic and reveals the evolution of ALS-FTD pathology. eLife 8:e39578. doi: 10.7554/eLife.39578

Zhang, Y., Gao, X., Saucedo, L. J., Ru, B., Edgar, B. A., and Pan, D. (2003). Rheb is a direct target of the tuberous sclerosis tumour suppressor proteins. Nat. Cell Biol. 5, 578-581. doi: 10.1038/ncb999

Zhang, C. H., Liu, H., Zhao, W. L., Zhao, W. X., Zhou, H. M., and Shao, R. G. (2021). G3BP1 promotes human breast cancer cell proliferation through coordinating with GSK- $3 \beta$ and stabilizing $\beta$-catenin. Acta Pharmacol. Sin. doi: 10.1038/s41401-020-00598-w [Epub ahead of print].

Conflict of Interest: The authors declare that the research was conducted in the absence of any commercial or financial relationships that could be construed as a potential conflict of interest.

Publisher's Note: All claims expressed in this article are solely those of the authors and do not necessarily represent those of their affiliated organizations, or those of the publisher, the editors and the reviewers. Any product that may be evaluated in this article, or claim that may be made by its manufacturer, is not guaranteed or endorsed by the publisher.

Copyright (c) 2021 Rehbein, Prentzell, Cadena Sandoval, Heberle, Henske, Opitz and Thedieck. This is an open-access article distributed under the terms of the Creative Commons Attribution License (CC BY). The use, distribution or reproduction in other forums is permitted, provided the original author(s) and the copyright owner(s) are credited and that the original publication in this journal is cited, in accordance with accepted academic practice. No use, distribution or reproduction is permitted which does not comply with these terms. 